\title{
Horizontal viscoelastic-gravitational displacement due to a rectangular dipping thrust fault in a layered Earth model
}

\author{
José Fernández \\ Instituto de Astronomía y Geodesia, Facultad de Ciencias Matemáticas, Ciudad Universitaria, Madrid, Spain
}

Ting-To $\mathrm{Yu}^{1}$ and John B. Rundle

Cooperative Institute for Research in Environmental Sciences, University of Colorado, Boulder

\begin{abstract}
Calculations of horizontal displacements due to a rectangular finite thrust fault in a viscoelastic-gravitational layered Earth model are presented. The Earth model consists of a single elastic-gravitational layer overlying a viscoelastic-gravitational half-space. A review of the full threedimensional theoretical solutions is presented along with the explicit solutions for horizontal displacements. Several examples of computations for dipping faults with various angles, and located at different depths, are shown. The results indicate that viscoelasticity introduces a long-wavelength component into the interseismic deformation field which is not present in published elastic techniques and also that a proper consideration of gravity is necessary only for near-field computations at longer periods of time. A pattern is found in the cumulative displacement of cycled earthquakes, which indicates that the viscoelastic displacements are visible for longer recurrence time events and that these may serve as a time index for the various stages between cycles.
\end{abstract}

\section{Introduction}

One important goal in crustal deformation research is to understand the postseismic deformation following large earthquakes. High-precision data obtained by modem land- and space-based instrumentation provide a feasible monitoring technique for such deformation processes, especially for horizontal movements.

Nur and Mavko [1974], Smith [1974], Bischke [1974], Rundle [1978], Thatcher and Rundle [1979], Thatcher et al., [1980] and Matsu'ura and Tanimoto [1980] focused on explaining crustal motions in areas of thrust faulting. In most of the previous work the omission of gravitational effects is justified [Rumdle, 1982a] because only deformation for the short time periods following earthquakes was modeled. The gravitational effect must be included for deformations that involve longer periods of time, for the reason that gravity will affect both the magnitude and pattern at longer periods of time, as shown in the following plots.

Thatcher and Rundle [1979] and Rundle and Thatcher [1980] have made use of inelastic displacement models to explain patterns of crustal deformation based on a numerical technique developed by Rundle [1978]. This method enables one to calculate surface displacements occurring after the insertion of a displacement into a medium consisting of an elastic layer over a linear, viscoelastic half-space.

The two principal defects in the work cited above were the lack of inclusion of gravitational effects in the calculations and the inability to calculate viscoelastic displacements for long periods of time afterward (tens to hundreds of Maxwell times). For purely elastic calculations, gravitational effects manifest themselves over distances greater than $1000 \mathrm{~km}$ and thus have little relevance to

\footnotetext{
Now at Institute of Earth Sciences, Academia Sinica, Taipei, Taiwan.
}

Copyright 1996 by the American Geophysical Union.

Paper number $96 \mathrm{JB} 00525$.

0148-0227/96/96JB-00525\$09.00 deformation in the near-source region However, this is not the case for viscoelastic calculations [Rundle, 1981a, b], and for viscoelastic-gravitational deformation the inclusion of gravity will affect the postseismic displacement field even in the vicinity of the fault [Yu, 1995].

A series of recent calculations have examined various aspects of this problem Ma and Kusznir [1992, 1993, 1994] simulate multilayered relaxed viscoelastic gravitational models by setting the elastic Lame parameters in one or more layers to zero. No time dependence is examined. Cohen [1994] models the earthquake cycle in thrust domains using a plane-strain finite element calculation. Our present calculations extend these results to a systematic examination of three-dimensional thrust faulting in viscoelastic-gravitational models using the techniques developed by Rundle [1981a].

Rundle [1980] explicitly solved the coupled elasticgravitational field equations and derived the displacements resulting from the insertion of point sources in a layered halfspace. Rundle [1981a] obtained the solution of the coupled viscoelastic-gravitational problem These solutions generally proceed in three steps. First, the Green's functions for the elasticgravitational field equations are computed Next the correspondence principle that relates the elastic-gravitational solutions to the Laplace-transformed viscoelastic-gravitational solutions is applied. Upon completion of the inverse transform into the time domain, the Green's function is integrated over the finite source region to obtain the time-dependent near-field displacements. Rundle [1981a] collected the necessary numerical techniques and produced calculations of the elastic dip-slip Green's functions for vertical deformation. He found that as $\mu$ the half-space rigidity, decreases in magnitude, the absolute effects of gravity become more important. This property implies that for the time-dependent displacements which are the result of faulting in a viscoelastic medium, the effects of gravity will only become important over sufficiently long time intervals, e.g., when $\mu$ has relaxed to some small value. 
Rundle [1981a] compares the relative effects of nonzero value of $G$, the gravitational constant, with $g$, surface acceleration due to gravity, in modifying the purely elastic surface displacement. Rundle [1982a] computes vertical displacements due to thrust faulting in a viscoelastic-gravitational layered Earth model and compares them to data from the south Kanto region of Japan. Time-dependent horizontal deformations were not computed by this technique, and high-precision observations of horizontal deformation using space geodetic techniques make the need for such a computation essential.

In the next sections we present a brief review of the theoretical method outlined by Rundle [1980, 1981b, 1982a] and the extension to horizontal viscoelastic-gravitational displacements due to a rectangular thrust fault. We then conclude with some examples of theoretical computations.

\section{Model}

Rundle [1980] solves the elastic-gravitational problem for a thrust fault in a layered elastic medium. Green's functions, which are the response of a point source for a layered medium, are computed using propagator matrices [Thomson, 1950; Haskell, 1953; Gilbert and Backus, 1966] and source functions located at an appropriate source depth. The necessary numerical techniques were collected by Rundle [1981a], and calculations of the elastic dip-slip Green's functions for vertical deformation were carried out. One of the major difficulties encountered in that work was the existence of poles in the integration of the Green's function along the strike and dip of the fault plane. The integration over the poles, when they exist, can be accomplished using a simple technique [Longman, 1960]. We have found that these poles play an insignificant role in the solution and the speed of calculation can be increased considerably by setting the kernels equal to zero at these locations.

Rundle [1981a] found that for displacements due to dip-slip faulting in a layered elastic-gravitational medium, self-gravitation effects arising from the nonzero value of $G$ were generally much smaller than effects related to the surface gravitational acceleration $\boldsymbol{g}$ (the "reduced problem"). We present a short summary of this problem and its solution as given by Rundle [1981a].

\section{Reduced Problem}

This result is implemented by Rundle [1981b] by considering the governing equations [Love, 1911; Rundle, 1980] and setting
$G=0$, which implies that $\phi$, the gravitational potential, is constant and gives as a result the following vector equation for an infinite space:

$$
\Delta^{2} \underline{u}+\frac{1}{1-2 \sigma} \nabla \nabla \cdot \underline{u}+\frac{\rho_{0} g}{\mu} \nabla\left(\underline{u} \cdot \underline{e}_{z}\right)-\frac{\rho_{0} g}{\mu} \underline{e}_{z} \nabla \cdot \underline{u}=0
$$

Here $\underline{u}$ is the perturbed displacement in the deformed cylindrical coordinate system $(r, \theta, z)$, and $\underline{e}_{z}$ is the orthogonal unit vector in the $z$ direction. The method and technique used to solve equation (1) and to obtain the corresponding solution for a layered medium are outlined in detail by Rundle [1980].

We shall be concerned with an Earth model consisting of one layer over a half-space. The layer, thickness $H$, has elasticgravitational properties. The half-space has viscoelasticgravitational properties and can relieve imposed stresses by flowing in response. We shall refer to the layer properties as $\lambda_{Z}$ $\mu_{L} \rho_{L}$ and the half-space properties as $\lambda_{\oiint} \mu_{\oiint} \rho_{H}$. With these definitions, we write the elastic-gravitational displacements at the surface $z=0$ due to a dipping, rectangular thrust fault of length along strike $2 L$, with downdip width $W$ and dip angle $\psi$ (Figure 1) as

$$
\begin{gathered}
\underline{u}=\frac{H(t)}{4 \pi} \int_{0}^{w} d \xi \int_{-L}^{L} d \zeta U(\xi, \varsigma) \int_{0}^{\infty}\left\{\left(x_{0}^{1}(0) \underline{P}_{0}+x_{2}^{1}(0) \underline{P}_{2}\right.\right. \\
\left.+y_{0}^{1}(0) \underline{B}_{0}+y_{2}^{1}(0) \underline{B}_{2}+z_{2}^{1}(0) \underline{C}_{2}\right) \sin 2 \psi \\
\left.-\left(x_{1}^{1}(0) \underline{P}_{1}+y_{1}^{1}(0) \underline{B}_{1}+z_{1}^{1}(0) \underline{C}_{1}\right) \cos 2 \psi\right\} k d k
\end{gathered}
$$

where

$$
\begin{aligned}
& \underline{P}_{m}=J_{m}(k r) e^{i m \theta} \underline{e}_{z} \\
& \underline{B}_{m}=\left[\frac{\partial J_{m}(k r)}{\partial k r} \underline{e}_{r}+i m \frac{J_{m}(k r)}{\partial k r} \underline{e}_{\theta}\right] e^{i m \theta} \\
& \underline{C}_{m}=\left[i m \frac{J_{m}(k r)}{\partial k r} \underline{e}_{r}-\frac{\partial J_{m}(k r)}{\partial k r} \underline{e}_{\theta}\right] e^{i m \theta}
\end{aligned}
$$

where $\xi$ is an integration coordinate downdip, $\zeta$ is a coordinate along strike, $J_{m}(k r)$ are cylindrical Bessel functions of order $m$

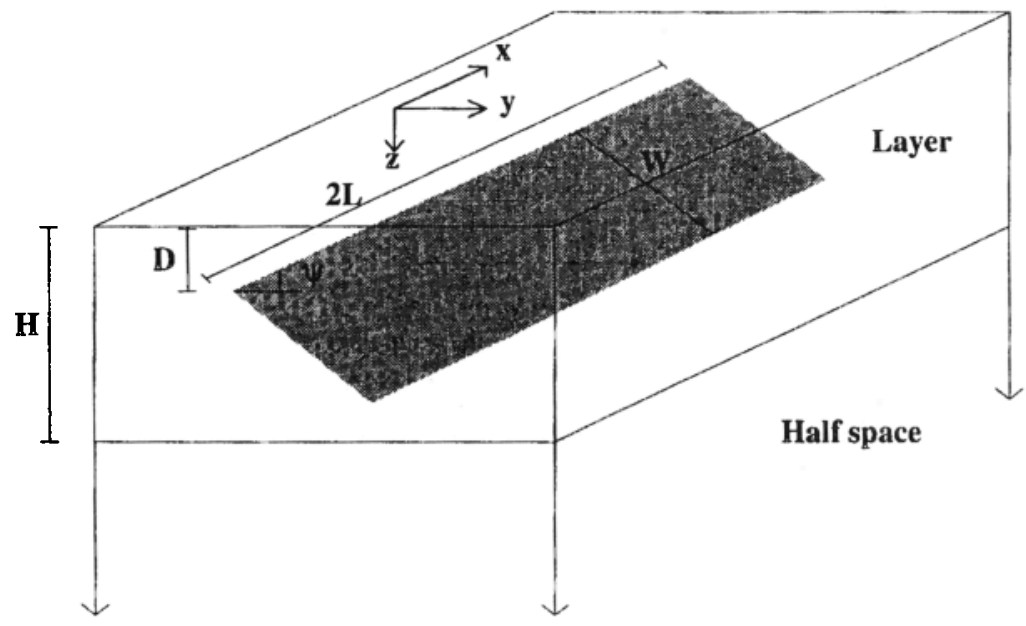

Figure 1. Geometry and coordinate system for a rectangular, finite, dipping fault in elastic-gravitational layer over a viscoelastic-gravitational half-space. $D$ is depth, $W$ is fault plane width, $2 L$ is length along strike, $\Psi$ is dip angle, and $H$ is layer thickness [after Rundle, 1982a]. 

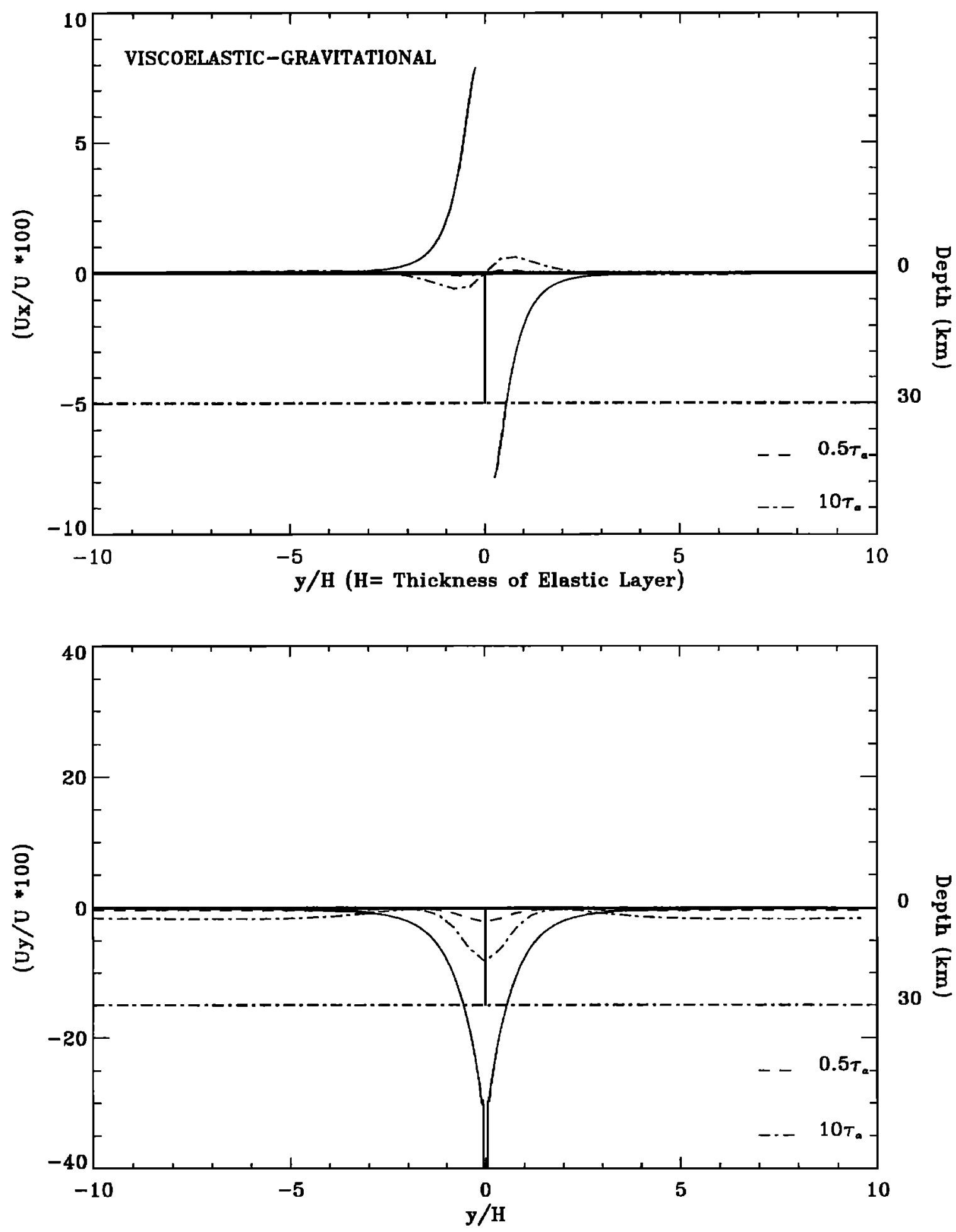

Figure 2. Surface horizontal $\left(U_{x}\right.$ and $\left.U_{y}\right)$ displacements due to a $90^{\circ}$ dipping thrust fault in an elastic-gravitational layer over a viscoelastic-gravitational half-space at $X=10 \mathrm{~km}$. Fault is $2 L=30 \mathrm{~km}$ long, $D=0 \mathrm{~km}, W=30 \mathrm{~km}$, and $H=30 \mathrm{~km}$. The coordinates at the left show the ratio of induced displacement to displacement on the fault and the right axis shows the thickness of the elastic layer. Horizontal thick lines represent the surface and the vertical/inclined thick line is the fault, the thick dashed line is the boundary of elastic layer and half-space. The solid curves are the coseismic displacements, the short-dashed curves are the $0.5 \tau_{a}$ postseismic displacements, and the longest dash curves are the $10 \tau_{a}$ postseismic displacements. 


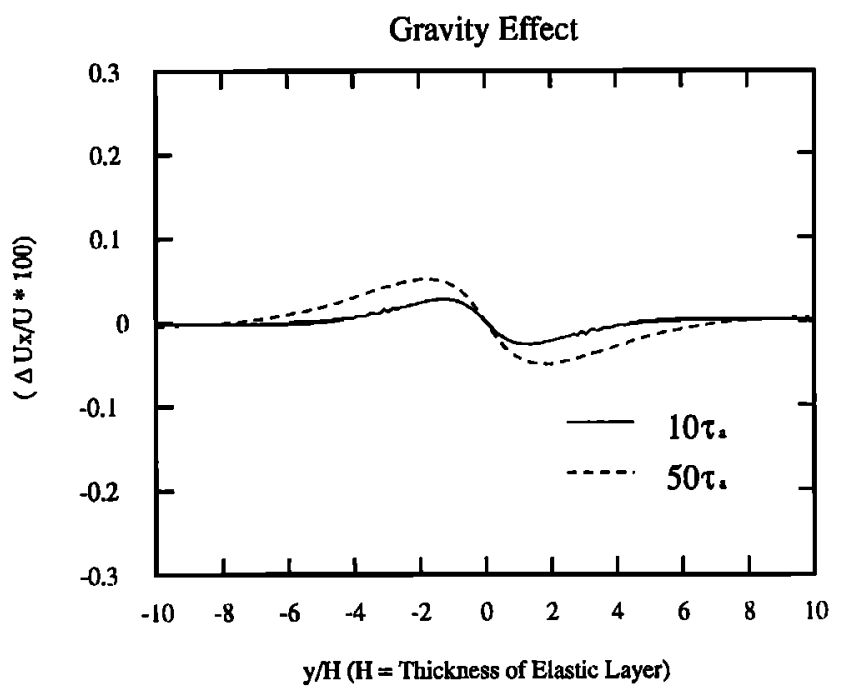

$$
\begin{gathered}
\varepsilon_{3}=-\left(y_{2}^{1}(0) \frac{J_{1}(k r)+J_{3}(k r)}{2}\right. \\
\left.-z_{2}^{1}(0) \frac{J_{1}(k r)-J_{3}(k r)}{2}\right) \sin \theta \\
\varepsilon_{4}=-\left(y_{1}^{1}(0) \frac{J_{0}(k r)+J_{2}(k r)}{2}\right. \\
\left.+z_{1}^{1}(0) \frac{J_{0}(k r)-J_{2}(k r)}{2}\right) \cos \theta
\end{gathered}
$$

and using them we have

$$
\begin{gathered}
\underline{u}_{x}=\frac{H(t)}{4 \pi} \int_{0}^{w} d \xi \int_{-L}^{L} d \zeta U(\xi, \zeta) \int_{0}^{\infty}\left\{\left(\varepsilon_{1} \sin 2 \psi+\varepsilon_{2} \cos 2 \psi\right) \cos \theta\right. \\
\left.+\left(\varepsilon_{3} \sin 2 \psi-\varepsilon_{4} \cos 2 \psi\right) \sin \theta\right\} k d k \\
\underline{u}_{y}=\frac{H(t)}{4 \pi} \int_{0}^{w} d \xi \int_{-L}^{L} d \zeta U(\xi, \varsigma) \int_{0}^{\infty}\left\{\left(\varepsilon_{1} \sin 2 \psi+\varepsilon_{2} \cos 2 \psi\right) \sin \theta\right. \\
\left.-\left(\varepsilon_{3} \sin 2 \psi-\varepsilon_{4} \cos 2 \psi\right) \cos \theta\right\} k d k
\end{gathered}
$$

The necessary source functions are given by Rundle [1980] in his equations (97)-(103) and equation (109) and by equations (11) and (12) of Rundle [1982b].

\section{Introduction of Time Dependence}

The introduction of time dependence into equations (8) and (9) is done by following Rundle [1982a]. As a first step, the comespondence principle of linear viscoelasticity is applied [Fung, 1965]. This requires that the elastic quantities $\lambda$ and $\mu$ in each component of the elastic solution (from now on represented by $u(t))$ be replaced by their Laplace-transformed quantities $s \bar{\lambda}$ and $s \bar{\mu}$ to obtain $\vec{u}(s)$, where the bar signifies the Laplace transformed quantity and $s$ is the parameter conjugate to time, and $\bar{u}(s)$ is then inverted to give $u_{v}(t)$, the solution to the viscoelastic problem The specific method used to perform the inversion is the Prony series method, in which the function $u_{v}(l)$ is approximated by a function $u_{v}$ ( $(t)$ consisting of a series of decaying exponentials. This simple method is extremely cost-effective and can be performed fast and efficiently. Hence we set [Schapery, 1961; Cost, 1964]

$$
u_{v}(t) \cong \sum_{i j}^{N} A_{j} \tau_{j}\left(1-e^{-t / \tau_{i}}\right)=u_{v}^{*}(t)
$$

gritational acceleration $g_{0}$ and wave number $k$. $H(t)$, the Heaveside step, is the time-dependent part of the applied displacement, $U(\xi, \zeta)$.

Using (3) and well-known relations between Bessel functions, we obtain the expressions for the horizontal displacement in $x$ and $y$ coordinates. To do so, we define the quantities

$$
\begin{gathered}
\varepsilon_{1}=-\left[y_{0}^{l}(0) J_{1}(k r)+\left(z_{2}^{1}(0) \frac{J_{1}(k r)+J_{3}(k r)}{2}\right.\right. \\
\left.\left.-y_{2}^{l}(0) \frac{J_{1}(k r)-J_{3}(k r)}{2}\right) \cos 2 \theta\right] \\
\varepsilon_{2}=\left(y_{1}^{1}(0) \frac{J_{0}(k r)-J_{2}(k r)}{2}\right. \\
\left.+z_{2}^{1}(0) \frac{J_{0}(k r)+J_{2}(k r)}{2}\right) \sin 2 \theta
\end{gathered}
$$

where $\cong$ means "approximately equal, in the least squares sense", $\left\{\tau_{i}\right\}$ is a set of $N$ relaxation times and the $A_{i}$ are a set of unknown constants that can be determined by least squares methods. The set of relaxation times we use is $\left\{0.5 \tau_{\infty}, \tau_{a}, 5 \tau_{a}, 10 \tau_{a}, 50 \tau_{a,}, 100 \tau_{a}\right\}$. The asthenosphere relaxation time $\tau_{\mathrm{a}}$ is the characteristic time defined by $\tau_{a}=2 \eta / \mu_{\text {r }}$, where $\eta$ is the viscosity of the Maxwell fluid. This approximation method presents the advantage of smooth time domain results in the time interval required and involves as few function evaluations as possible. The error obtained by using this numerical technique is thus minimized and $u_{v}$ " $(t)$ can then be integrated over the source region to obtain the required solution.

\section{Results}

We show the horizontal displacement field due to a vertical thrust fault rupturing the entire elastic layer in Figure 2. It is found 

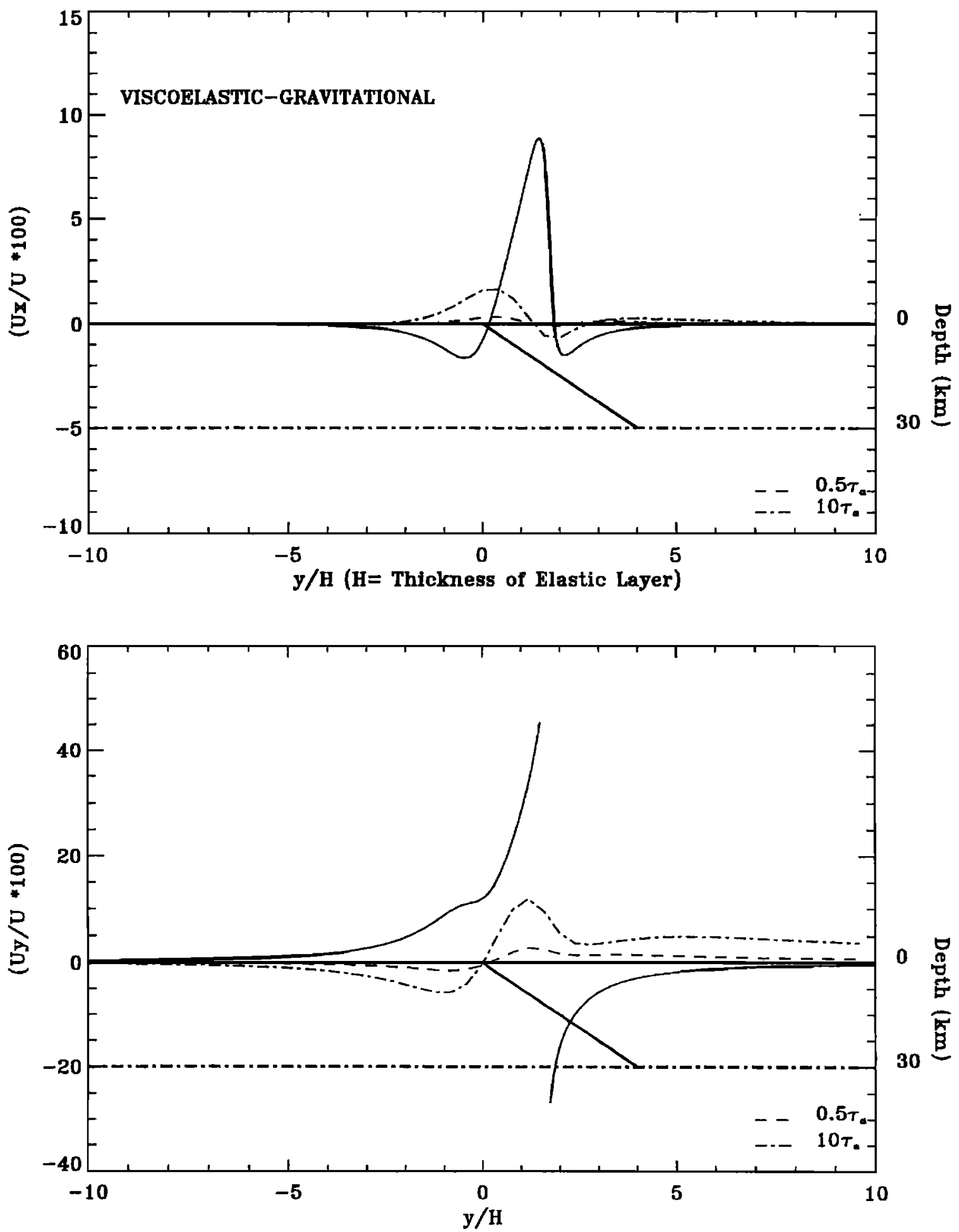

Figure 4. Surface horizontal displacements due to a $30^{\circ}$ dipping thrust fault in an elastic-gravitational layer over a viscoelastic-gravitational half-space at $X=10 \mathrm{~km}$. Fault is $2 L=30 \mathrm{~km}$ long, $D=0 \mathrm{~km}, W=60 \mathrm{~km}$, and $H=30 \mathrm{~km}$.

that the inclusion of gravity only affects the near-field $(y / H<10)$ postseismic displacements after longer periods of time. This can be seen clearly in Figure 3, which shows the differences between viscoelastic-gravitational and purely viscoelastic displacements for the same fault as in Figure 2. For the $50 \tau_{\mathrm{a}}$ curve in Figure 3 both postseismic $U_{x}$ and $U_{y}$ displacements at the fault are about $10 \%$ larger for purely viscoelastic calculations. This difference depends on the thickness and density of the elastic layer and also on the considered $X$ coordinate. The displacement parallel to the fault
$\left(U_{x}\right)$ has the least change in magnitude. It can be seen in Figure 2 that the postseismic displacements have a much longer wavelength than the coseismic elastic response. Melosh and Raefsky [1983] also obtain a similar pattem for $U_{y}$ displacements ( $U_{x}$ in their paper) in the far field using finite element calculations. This is the most distinct difference between the viscoelastic and other purely elastic models. The elastic model uses a downdip extension of slip to interpret the postseismic displacement [Fitch and Scholz, 1971; Savage, 1995], by treating Earth as a 
Gravity Effect
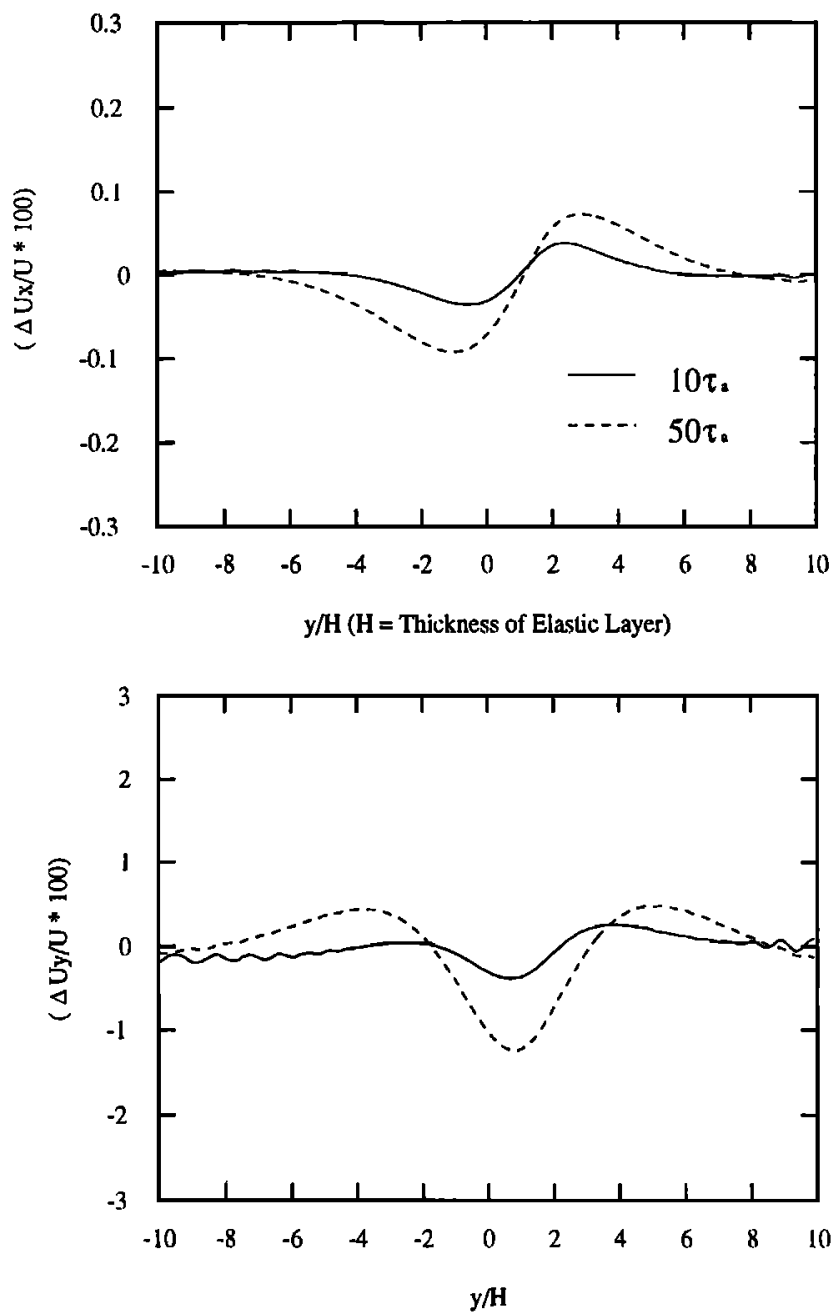

Figure 5. Viscoelastic-gravitational minus purely viscoelastic displacements for $X=10 \mathrm{~km}$ and the same fault as in Figure $4 ; 10$ and $50 \tau_{a}$ are considered in this figure to show clearly the differences that appear after long periods of time.

homogeneous elastic medium. They then compute the displacements due to the time-dependent postseismic downdip-extensionslip. This technique models the near-field amplitude by adjusting the amount of slip and matches the pattern of displacements by changing the dip angle. However, it only produces shortwavelength features in the near field for elastic media.

We next change the dip angle from vertical to $30^{\circ}$ and show the results in Figure 4 . It will be observed that $U_{y}$ has a very longwavelength postseismic displacement after a longer period of time. Figure 5 shows again the differences between viscoelasticgravitational and purely viscoelastic displacements for a fault with the same characteristics as in Figure 4. Again, the inclusion of gravity induces changes in magnitude of the displacements. $U_{y}$ decreases about $12 \%$ and $U_{x}$ changes only about $6 \%$ after tens of relaxation times in the near field.

Figures 6 to 9 show the displacement field caused by the fault rupturing only half the thickness of the elastic layer. First, we show a fault breaking only the upper half of the layer (Figure 6). Figure 6 shows a similar pattem to that in which the entire layer is ruptured (Figure 2) in both components. The magnitude of postseismic displacement $U_{x}$ is also quite similar, but $U_{y}$ displacements are smaller in the case of nupturing the top half of the layer (note the change in scale). Figure 7 shows a fault that only breaks the bottom half of the layer, here the magnitude of $U_{x}$ displacement has decreased and the discontinuity of the coseismic displacement at the surface trace of the fault has vanished. The magnitude of postseismic $U_{y}$ is about 1000 times larger than $U_{x}$ in this particular case for a point located close to the fault. The most distinct difference between the surface and the buried fault is the $U_{y}$ coseismic displacement field for $y / H<2$, the buried fault produces a " $W$ "-shaped displacement, while the surface fault results in a " $U$ " shape. This could be the key tool for identifing the ruptured depth of the fault when measuring the surface displacement field $U_{y}$ has a distinct "spike"-type postseismic displacement field in the vicinity of the fault zone and has a very long wavelength

We altered the dip angle from vertical to $30^{\circ}$ and let the fault rupture only the top and bottom half of the elastic layer in Figures 8 and 9, respectively. When the fault ruptures only the top half of the elastic layer, both the $U_{x}$ and $U_{y}$ displacements have similar pattern and magnitude (differences within 15 to 20\%) to a fault that ruptures the entire elastic layer (Figure 4). It can be seen in both Figures 4 and 8 that $U_{x}$ is different both in pattern and size from $U_{y}$ For a $30^{\circ}$ dipping fault that ruptures only the bottom half of the elastic layer, the magnitude of $U_{x}$ is similar to the magnitude of $U_{y}$ and the wavelength of postseismic $U_{x}$ displacement is less than that resulting from the surface fault. However, the $U_{y}$ pattern is different from, and its wavelength is greater than, that resulting from the surface fault. The distinct displacement features associated with each fault geometry provide a means to distinguish the parameters of the fault and will be reflected in inversions of the surface displacement field using leveling or Global Positioning System (GPS) data. The pattern and magnitude of the displacement field are most sensitive to the depth of the fault and location of the profile. For this reason, changing fault strike will create a larger diversity than changing dip angle in the process of matching the displacement field to a fault dislocation model.

All the profiles shown in Figures 2 to 9 have been computed for $X=10 \mathrm{~km}$ (1/3 length of the fault) instead of $X=0 \mathrm{~km}$ in order to study the variations with the characteristics of the fault (for symmetry arguments there can not be any fault-parallel displacements along a line perpendicular to the center of the fault).

We also carried out earthquake cycle computations using the method described by Cohen and Kramer [1984] to calculate the components of deformation $\left(U_{x}\right.$ and $\left.U_{y}\right)$ measured at time $t$, since an earthquake at $t=0$. These components can be written as

$$
u(t)=v t+u_{2}(t)+\sum_{m=1}^{\infty}\left[u_{2}(t+m T)-u_{2}(m T)\right]
$$

where $v t$ is the response to a steady block motion and $u_{2}(t)$ is the contribution to the crustal deformation from the coseismic slip and the aseismic "backslip" during the current earthquake cycle (see Figure 10). The last term is the viscoelastic dislocation contribution from all previous events $(m)$. This model represents offset of an elastic plate with constant velocity $(v)$, together with "backslip" to lock the fault, and a sequence of prior earthquakes having a uniform recurrence time interval $T$. The fault offset is uniform for each earthquake, and no movement is allowed between events. The remainder of the plate boundary is slipping steadily at the rate $v$. The latter condition is enforced by assigning a so called "backslip" of amount $-\nu$ in the fault zone to lock it. The plate boundary below the lithosphere is assumed to be 

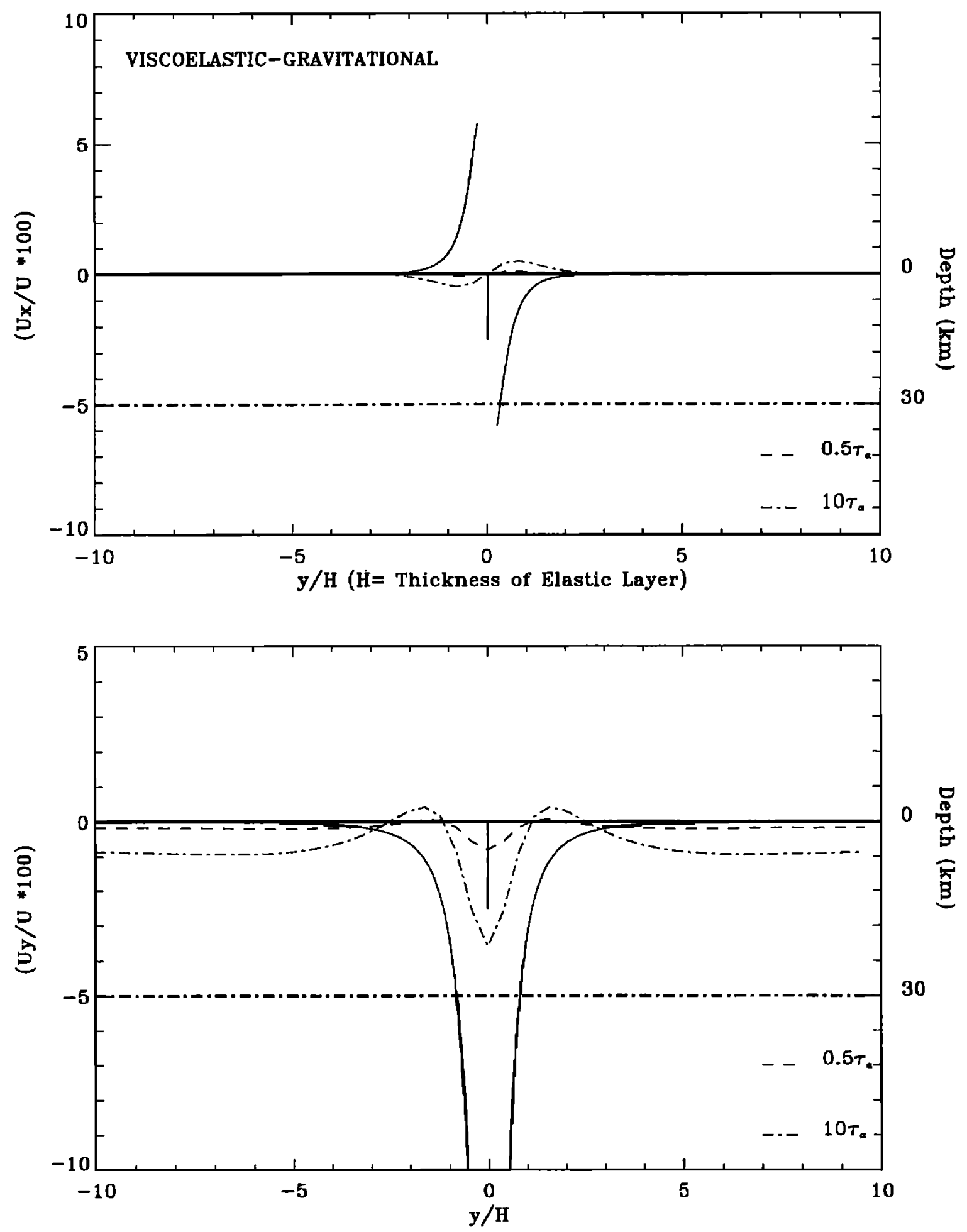

Figure 6. Surface horizontal displacements due to a $90^{\circ}$ dipping thrust fault in an elastic-gravitational layer over a viscoelastic-gravitational half-space at $X=10 \mathrm{~km}$. Fault is $2 L=30 \mathrm{~km}$ long, $D=0 \mathrm{~km}, W=15 \mathrm{~km}$, and $H=30 \mathrm{~km}$

subducting aseismically and to contribute no deformation at the surface additional to that produced by the rigid body plate kinematic contribution. The validity of this assumption has been verified by comparisons to two dimensional, plane strain finite element models of the subduction process [Rundle and Smith, 1982; S. C. Cohen, personal communication, 1988]. The prescription we follow in this model is, in general, valid for subduction zones, and this is in fact the major application for the results presented here.

In order to plot the viscoelastic displacement caused by the cyclic earthquakes during various time intervals, we set the recurrence time equal to $\tau_{a}, 10 \tau_{a}$ and $100 \tau_{a}$ in Figures 11,12 , and 13 , respectively. The plate velocity is $v=U / T$, where $U$ is the displacement at the fault for each earthquake. As expected, plate 

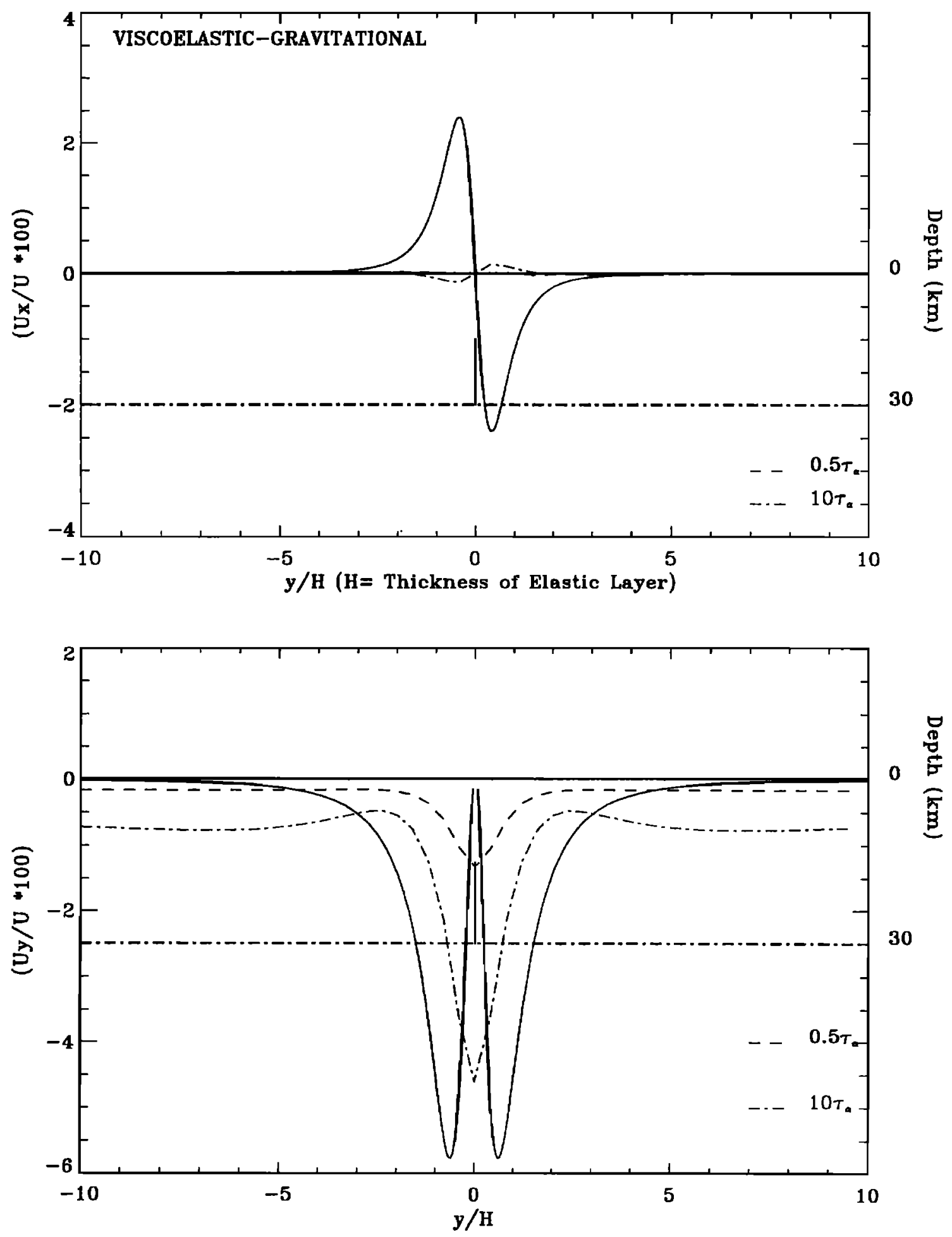

Figure 7. Surface horizontal displacements due to a $90^{\circ}$ dipping thrust fault in an elastic-gravitational layer over a viscoelastic-gravitational half-space at $X=10 \mathrm{~km}$. Fault is $2 L=30 \mathrm{~km}$ long, $D=15 \mathrm{~km}, W=15 \mathrm{~km}$, and $H=30 \mathrm{~km}$.

motion is the dominant deformation mode for all models with short recurrence time intervals (see Figure 11 ). With $T$ equal to 10 times the relaxation time, $\tau_{a}$ (see Figure 12), outward displacements (i.e., along the fault strike) were found near the fault in the early stage of the cycle $(t<0.67)$, caused by the change of viscoelastic displacements $U_{x}$ A long recurrence time case is examined by setting $T=100 \tau_{a}$. Here the magnitude of outward displacement decreased as time elapsed, but the distinct "looping" pattern toward the end of the fault can be easily found (see Figure 13). These displacement patterns can serve as an indicator for the various stages of a long recuntence interval thrust earthquake cycle.

\section{Summary and Conclusions}

We present an extension of a previous method to calculate the horizontal postseismic surface displacements in a layered medium. 

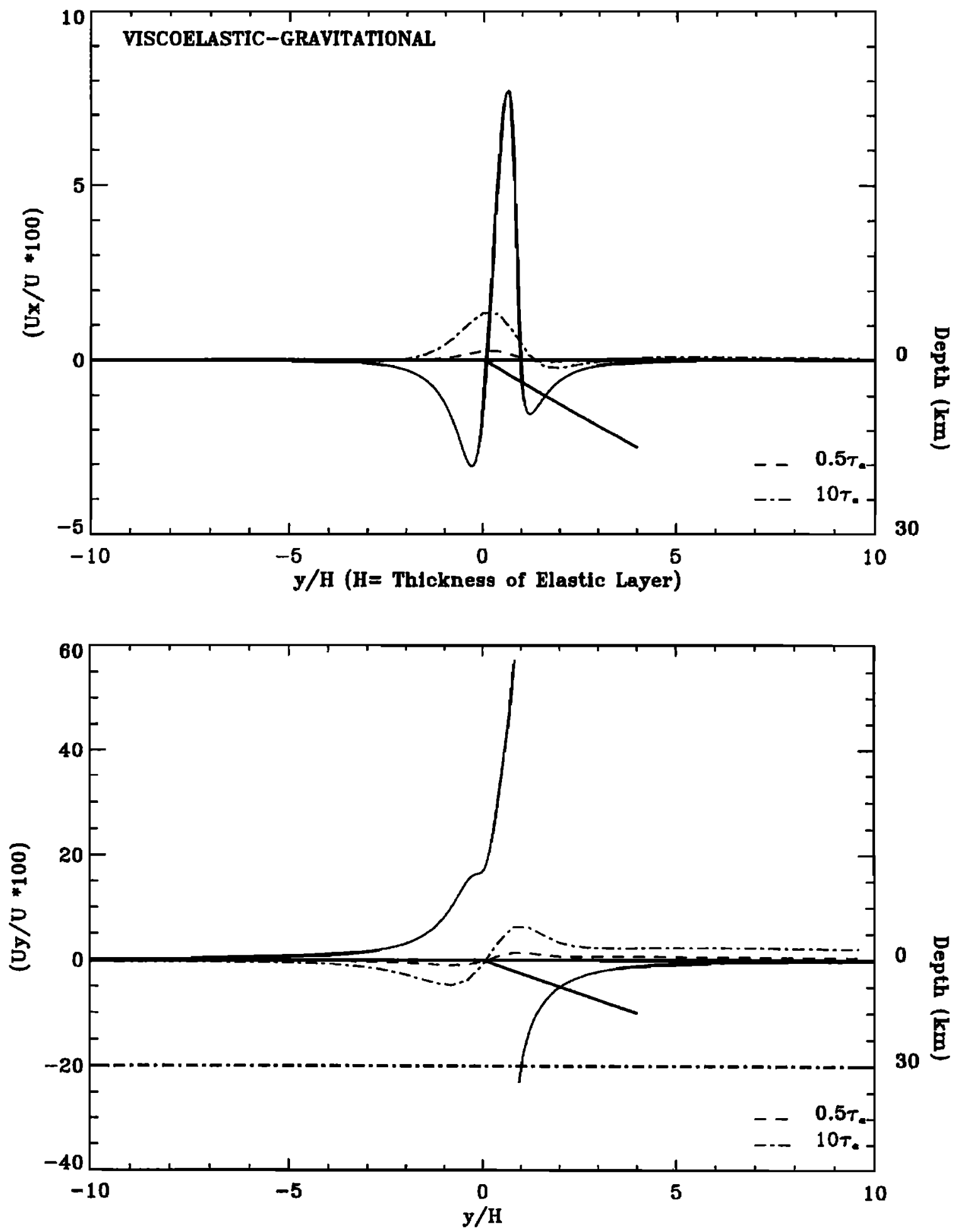

Figure 8. Surface horizontal displacements due to a $30^{\circ}$ dipping thrust fault in an elastic-gravitational layer over a viscoelastic-gravitational half-space at $X=10 \mathrm{~km}$. Fault is $2 L=30 \mathrm{~km}$ long, $D=0 \mathrm{~km}, W=30 \mathrm{~km}$, and $H=30 \mathrm{~km}$.

including gravitational effects in a layered medium composed of one elastic layer over a viscoelastic half-space. We find that gravity arrests changes in the displacement fields afler long periods of time. This is important in using the model to analyze high-precision data from space geodetic techniques.

It can be observed in the examples of computations shown in Figures 2 to 9 that $U_{y}$ is 4 times larger than $U_{x}$ for all the surface faults. Most of the displacement occurs in the $-2 H<y<2 H$ region. We also find that surface and buried faults have distinct features for both vertical and dipping faults.

Changing the downdip width of the fault and its minimum depth affects both the magnitude and pattern of all displacement fields, as found in previous work. It is possible to distinguish whether a fault extends through all of the elastic layer or only part of it from the pattern observed in the displacement field. It is found that postseismic $U_{y}$ (horizontal displacement perpendicular to the 

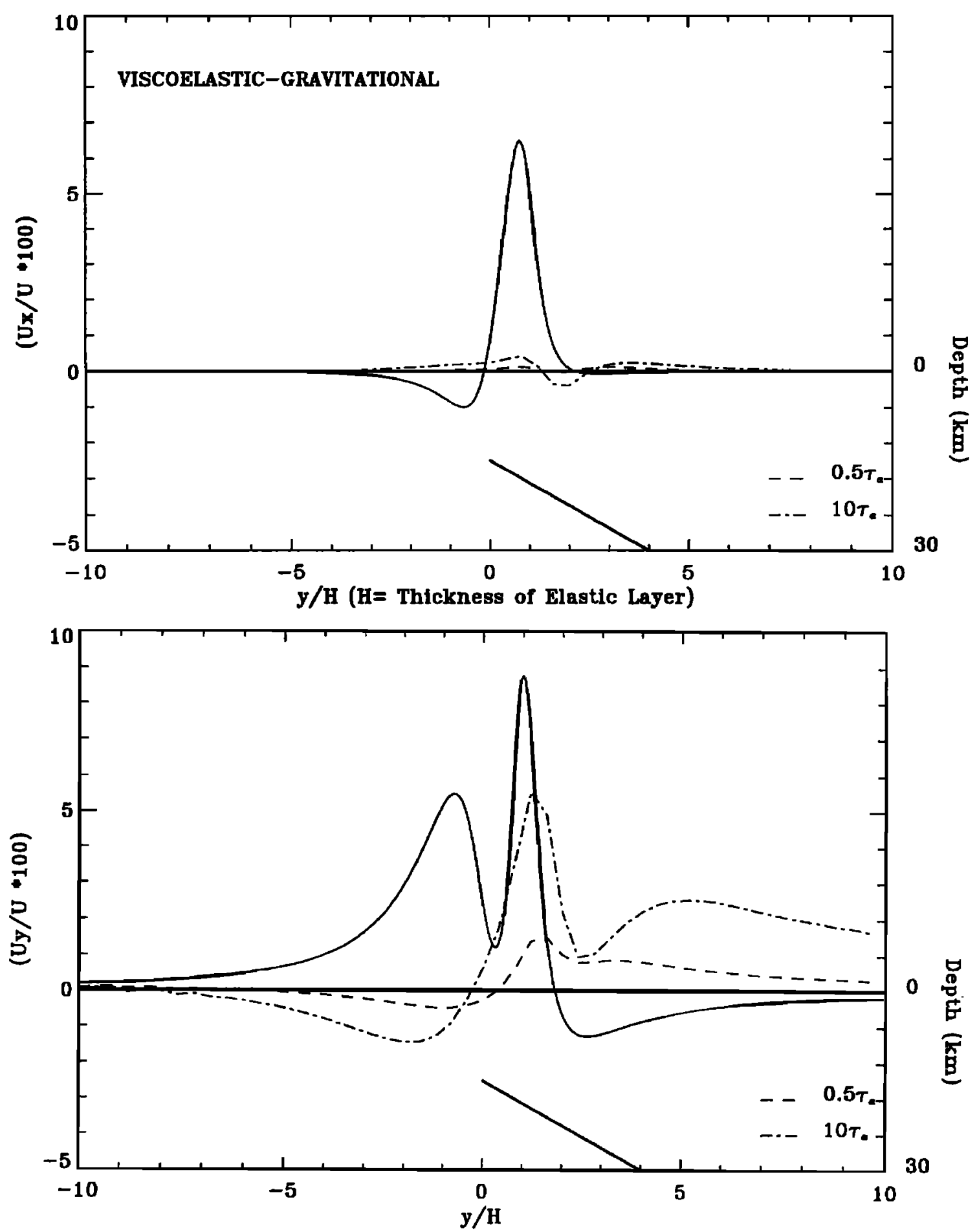

Figure 9. Surface horizontal displacements due to a $30^{\circ}$ dipping thrust fault in an elastic-gravitational layer over a viscoelastic-gravitational half-space at $X=10 \mathrm{~km}$. Fault is $2 L=30 \mathrm{~km}$ long, $D=15 \mathrm{~km}, W=30 \mathrm{~km}$, and $H=30 \mathrm{~km}$

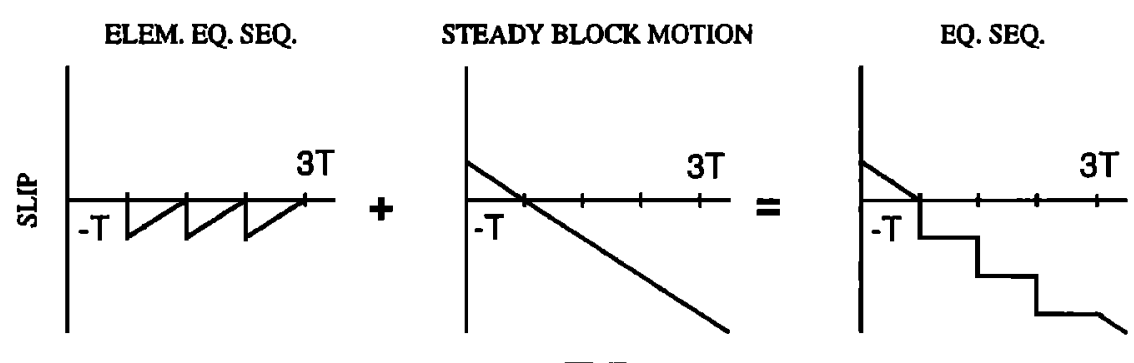

Figure 10. Diagram showing how an earthquake cycle (sequence) can be generated from superposition of an elementary cycle and steady block motion [after Savage and Prescott, 1978; Thatcher and Rundle, 1984]. 

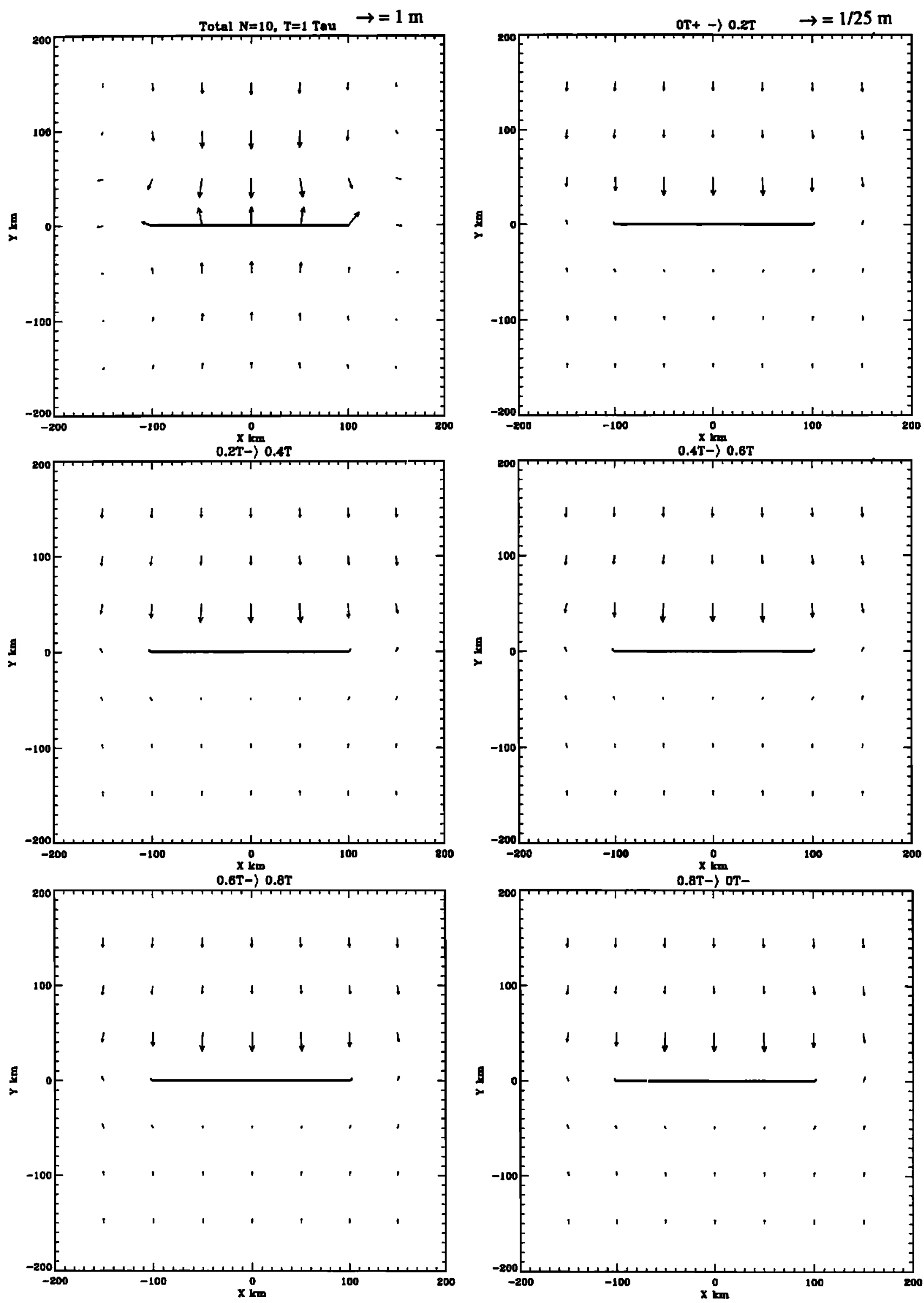

Figure 11. The accumulated displacement field due to 10 earthquakes prior to the event with recurrence time equal to 1 relaxation time. The thick line in the middle of the plot represents the fault. The coseismic displacement (top left) has its own scale. The rest of the charts use the same scale to represent the size of displacements. Except for the case of coseismic displacement, the size of the arrows is reduced by a factor of 25 . 

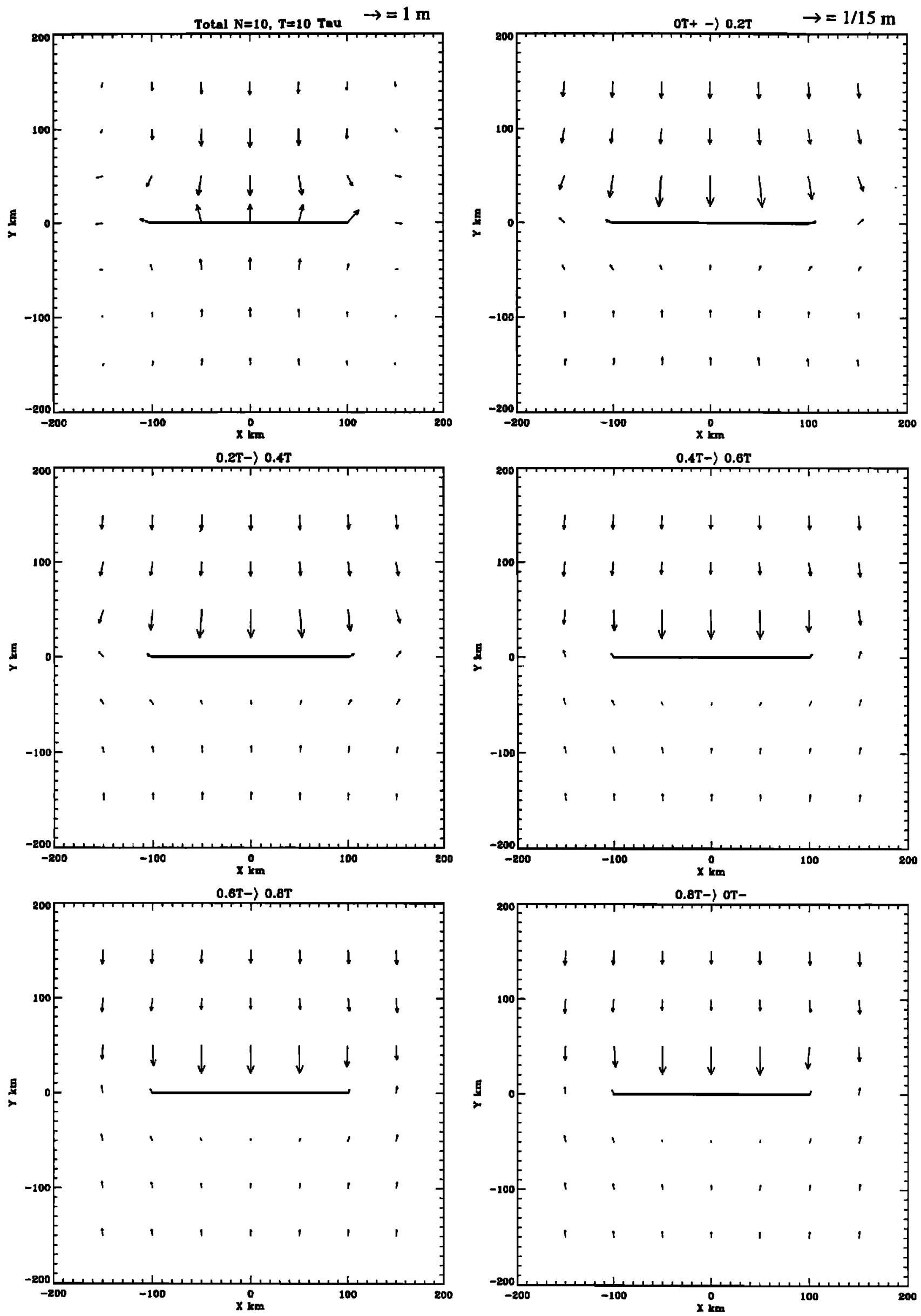

Figure 12. The same as for Figure 11, except that $T=10$ relaxation times and the size of the arrows for viscoelastic displacements are reduced by a factor of 15 . 

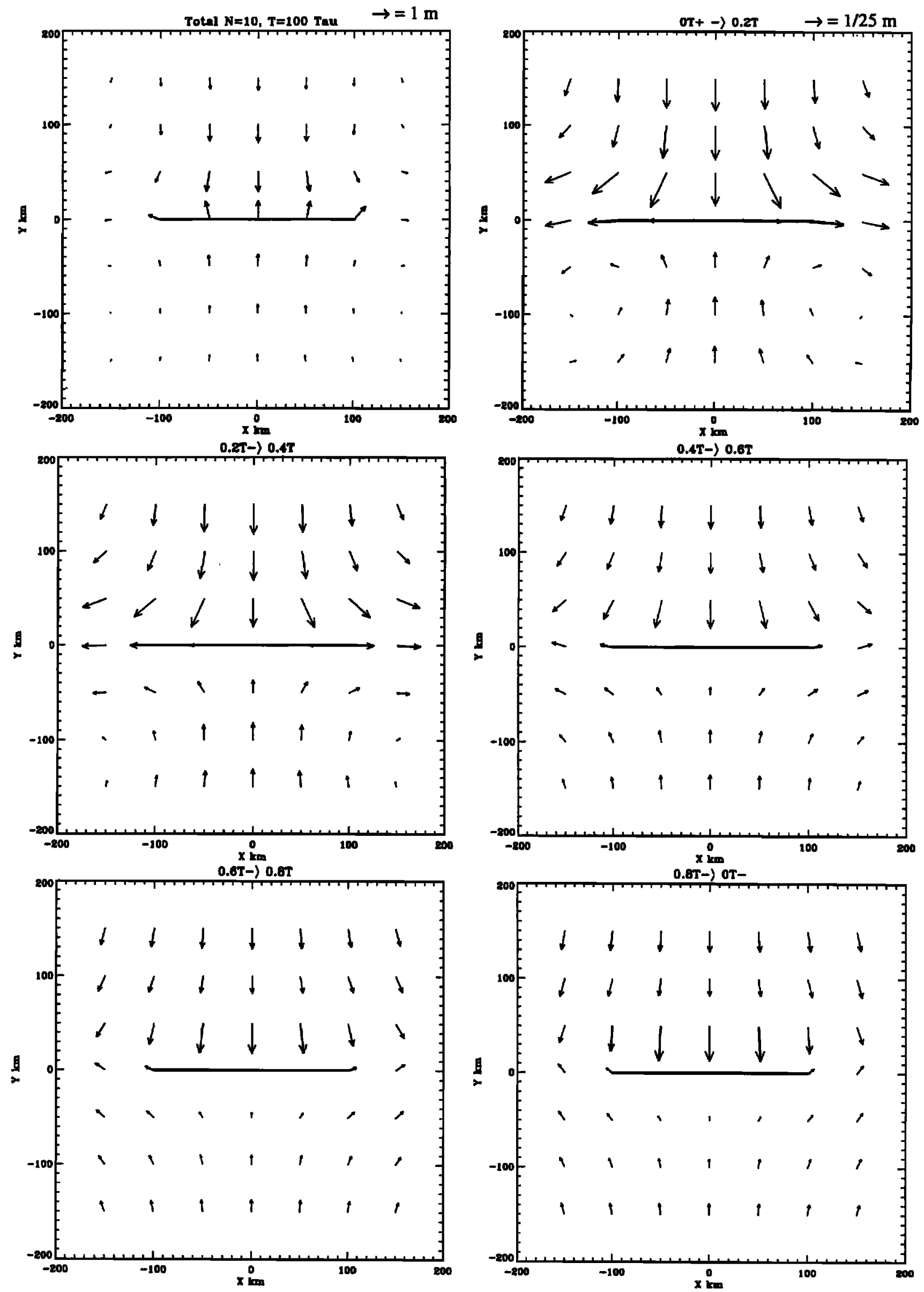

Figure 13. The same as for Figure 11 except that $T=100$ relaxation times. 
strike of the fault) is the dominant component, being about 100 to 1000 times $U_{x}$ when the fault breaks only the lower half of the elastic layer. The dip angle of the fault decides the ratio between these displacement components, which is another index for examining the fault geometry from displacement data. The numerical accuracy of this model is about $1 \%$ [Fernóndez and Rumdle, 1994; Yu, 1995] and is controlled by the precision of integrating the Green's function along the fault strike and dip.

The most important feature in the model is the long-wavelength postseismic displacement field, and this cannot be found in purely elastic models. The results of Cohen [1980] and Wahr and Wyss [1980] using finite element methods have the same character found with our analytic method. Moreover, the inclusion of gravity is necessary to properly describe the time dependence of the displacement field. We observe also that the magnitude and wavelength of postseismic displacements grow as time elapses.

One of the most important applications of the model presented here is the detailed examination of the time-dependent deformation of many earthquake cycles on the same fault plane. For models with a short recurrence time interval, the plate motion dominates the displacement field during the entire cycle, and major effects of the viscoelastic displacement only occur near the edge of the fault plane. However, for very long recurrence times, the accumulated viscoelastic displacement dominates the deformation field following the earthquake at large distances from the fault.

Acknowledgments. The research of José Fernández was supported with funds from project EVSV-CT92-0173 of the European Union in the framework of the Environment Program and by the Consejeria de Educacion y Cultura of the Comunidad de Madrid, which provided funds for his stay at CIRES, University of Colorado, Boulder. The research of John B. Rundle and Ting-To Yu has been supported by NASA grant NAG5-2353. We thank Michael Kozuch for reading the manuscript. We thank Andrea Donnellan and one anonymous reviewer who reviewed our paper and gave us useful comments to improve the quality of this paper.

\section{References}

Bischke, R.E. A model of convergent plate margins based on the recent tectonics of Shikoku, Japan, J. Geophys. Res., 79, 4845-4857, 1974.

Cohen, S.C., Postseismic viscoelastic surface deformation and stress, 1, Theoretical considerations, displacement, and strain calculations, J. Geophys. Res., 85, 3131-3150, 1980.

Cohen, S. C., Evaluation of the importance of model features for cyclic deformation due to dip-slip faulting, Geophys. J. Int., 119, 831846, 1994.

Cohen, S. C., and M. J. Kramer, Crustal deformation, the earthquake cycle, and models of viscoelastic flow in the asthenosphere, Geophys. J. R. Astron. Soc., 78, 735-750, 1984.

Cost, T.L., Approximate Laplace transform inversions in viscoelastic stress analysis, ALAA J., 2, 2157-2166, 1964.

Fernández, J, and J. B. Rundle, Gravity changes and deformation due to a magmatic intrusion in a two-layered crustal model, J. Geophys. Res., 99, 2737-2746, 1994.

Fitch, TJ., and C.H. Scholz, Mechanism of underthrusting in southeast Japan: A model of convergent plate interactions, J. Geophys. Res., 76, 7260-7279, 1971.

Fung Y.C, Fonondations of Solid Mechanics, Prentice-Hall, Englewood Cliffs, N.J., 1965.

Gilbert, F, and G. Backus, Propagator matrices in elastic wave and vibration problems, Geophusics, 31, 326-332, 1966.

Haskell, N.A. The dispersion of surface waves on multilayered media, Bull. Seismol. Soc. Am, 43, 421440, 1953.

Longman, I.M, A technique for the numerical solution of geophysical problems, J. Geophys. Res., 83, 5937-5945, 1960.
Love, A.E.H, Some Problems in Geodynamics, 180 pp., Cambridge Univ. Press, New York, 1911.

Ma, XQ. and N.J. Kusznir, 3-D subsurface displacement and strain fields for faults and fault arrays in a layered elastic half-space, Geophys. $J$. Int., $111,542-558,1992$.

Ma, X.Q. and N.J. Kusznir, Modeling of near-field subsurface displace ments for generalized faults and fault arrays, J. Struct. Geol., 15, 14711484, 1993.

$\mathrm{Ma}, \mathrm{XQ}$. and N.J. Kusmir, Effects of rigidity layering gravity and stress relaxation on 3-D subsurface fault displacement fields, Geophys. $J$. Int., 118, 201-220, 1994.

Matsu'ura, M., and T. Tanimoto, Quasi-static deformations due to an inclined, rectangular fault in a viscoelastic half-space, $J$. Phys. Earth, 28, 103-118, 1980.

Melosh, H.J, and A. Raefsky, Anelastic Response of the Earth to a dip slip erathquake, J. Geophys. Res., 88, 515-526, 1983.

Nur, A, and G. Mavko, Postseismic viscoelastic rebound, Science, 183, 204-206, 1974.

Rundle, J.B., Viscoelastic crustal deformation of a layered half-space by point couple source, J. Geophys. Res., 83, 5937-5945, 1978.

Rundle, J.B., Static elastic-gravitational deformation of a layered half-space by point couple sources, J. Geophys. Res., 85, 5355-5363, 1980.

Rundle, J.B, Numerical evaluation of static elastic gravitational deformation in a layered half-space by point couple sources, Rep. 81-2058, Sandia Natl. Lab., Albuquerque, N.M., 1981a.

Rundle, J.B., Vertical displacements from a rectangular fault in layered elastic-gravitational media, J. Phys. Earth, 29, 173-186, 1981 b.

Rundle, J.B., Viscoelastic-gravitational deformation by a rectangular thrust fault in a layered Earth, J. Geophys. Res., 87, 7787-7796, 1982a.

Rundle, J.B., Deformation, gravity, and potential changes due to volcanic loading of the crust, J. Geophys. Res, 87, 10729-10744, 1982b.

Rundle, J.B, and A.T. Smith, Comment on "Interpretation of postseismic deformation with a viscoelastic relaxation model, by $\mathrm{J}$. Wahr and M. Wyss", J. Geophys. Res., 87, 1079-1080, 1982.

Rundle, J.B., and W. Thatcher, Speculations on the nature of the Palmdale uplift, Bull. Seismol. Soc. Am, 70, 1869-1886, 1980.

Savage, J.C. Interseismic uplift at the Nankai subduction zone, southwest Japan, 1951-1990, J. Geoplyys. Res., 100, 6339-6350, 1995.

Savage, J.C., and W.H. Prescott, Asthenosphere readjustment and the earthquake cycle, J. Geophys. Res., 83, 3369-3376, 1978.

Schapery, RA, Approximate methods of transform inversion for viscoelastic stress analysis, Proc. U.S. Natl. Congr. Appl. Mech, 4th, 10751085,1961 .

Smith, A.T., Time dependent strain accumulation and relaxes at Island Ares: Implications for the 1946 Nankaido Earthquake, $\mathrm{Ph} . \mathrm{D}$. dissertation, Mass. Inst. of Technol., Cambridge, 1974.

Thatcher, W., and J.B. Rundle, A model for the earthquake cycle in underthrust zones, J. Geophys. Res., 84, 5540-5556, 1979.

Thatcher, W., and J.B. Rundle, A viscoelastic coupling model for the cyclic deformation due to periodically repeated earthquakes at subdution zones, J. Geophys. Res., 89, 7631-7640, 1984.

Thatcher, W., T. Matsuda, T. Kato, and J.B. Rundle, Lithospheric loading by the 1896 Riku-u earthquake, northern Japan: Implications for plate flexure and asthenospheric rheology, J. Geophys. Res., 85, 6429-6435, 1980.

Thomson, W.T., Transmission of elastic waves throughout a stratified medium, J. Appl. Phys., 21, 89-93, 1950.

Wahr, J., and M. Wyss, Interpretation of postseismic deformation with a viscoelastic relaxation model, J. Geophys. Res., 85, 6471-6477, 1980.

Yu, T. T., Crustal deformation due to dipping fault in elastic gravitational layer overlying viscoelastic gravitational half space: Models and applications, Ph.D. dissertation, Univ. of Colo., Boulder, 1995.

J. Fernánder, Instituto de Astronomía y Geodesia (CSIC-UCM), Facultad de Ciencias Matemáticas, Ciudad Universitaria, 28040-Madrid, Spain. (e-mail: pepe@sunas1 .mat.ucm.es)

J. B. Rundle, CIRES, University of Colorado, Boulder, CO 80309. (email: rundle@hopfield.colorado.edu)

T.T. Yu, Institute of Earth Sciences, Academia Sinica, P.O. Box 155, Nankang, Taipei, Taiwan. (e-mail: yutt@earth.sinica.edu.tw)

(Received December 31, 1994; revised January 30, 1996; accepted February 6, 1996.) 